Pädiatrie und Pädologie 2015 · 50:3

DOI 10.1007/s00608-015-0234-y

o) Springer-Verlag Wien 2015

\section{Wolfgang Sperl}

Vorstand der Univ.-Klinik für Kinder- und Jugendheilkunde SALK und

Präsident der Österreichischen Gesellschaft für Kinder- und Jugenheilkunde

\title{
Einen guten Start!
}

Meine sehr geehrte Damen und Herren, liebe Kolleginnen und Kollegen,

„Pädiatrie und Pädologie“ hat mich als neuen Präsidenten der ÖGKJ eingeladen, zu Heft 1/2015 ein Editorial zu verfassen, und ich bedanke mich dafür sehr herzlich.

„Pädiatrie und Pädologie“ ist zweifelsfrei die traditionsreichste österreichische Zeitung für Kinder- und Jugendheilkunde. Selbst habe ich in meine ersten Publikationen 1985 in „Pädiatrie und Pädologie“ verfasst, viele habilitierte Pädiater meiner Generation haben durch ihre Veröffentlichungen in „Pädiatrie und Pädologie" ihre wissenschaftliche Laufbahn begonnen und auch zur Positionierung der Österreichischen Pädiatrie beigetragen.

Mit der Entwicklung in der österreichischen Universitätslandschaft mit zunehmender Bedeutung und Wertung internationaler Vergleiche, der Wertung von Impactpunkten bei wissenschaftlicher Publikationen, der neuen Definitionen bzgl. wissenschaftlicher Qualifikation, wurden pädiatrische Wissenschaftler gedrängt, fast ausschließlich international zu publizieren. Damit hat der Wert von Päd \&Päd als wissenschaftliches Medium an Bedeutung verloren, trotzdem sind Fortbildungsinhalte und gesundheits -und standespolitisch wichtige Themen konstant erhalten geblieben.

Ich gratuliere Frau Dr. Höhl für die gelungene Akquise interessanter Beiträge, für die zuletzt sehr interessante und wichtige Entwicklung von „Pädiatrie und Pädologie“, mit bemerkenswerten Supplementen und Sonderausgaben, wie der Berichterstattung von der 7. Jahrestagung der politischen Kindermedizin mit dem Thema „Partizipation“ aber auch jetzt das letzte erschienene Supplement „Eltern unbekannt, Auswirkung auf die Identitätsfindung“. Ich bedanke mich auch beim Springer Verlag dafür. Aus meiner Sicht ist das die richtige Richtung. „Pädiatrie und Pädologie“ wird wiederum spezifisch für unsere österrei-

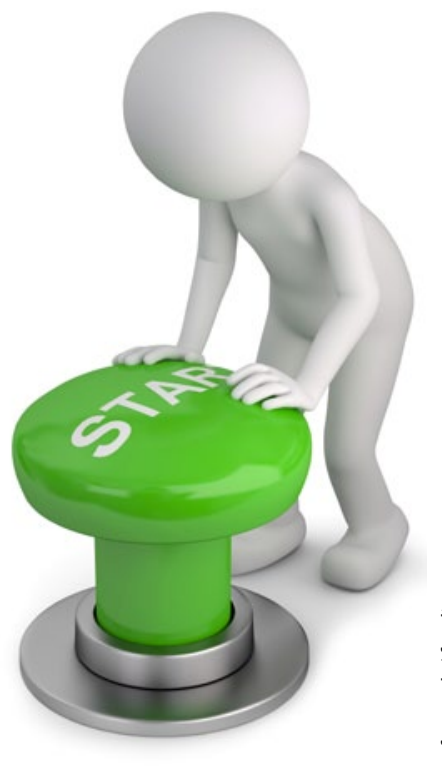

chische Kinder- und Jugendmedizin gerade in diesem Segment ein wichtiges Medium, relevante Inhalte gut $\mathrm{zu}$ transportieren. Auch gerade dem Thema „Schwerpunktsetzung in Österreich“ hat sich „Pädiatrie und Pädologie“ gewidmet.

Danke auch an Reinhold Kerbl, der die Schriftleitung in „Pädiatrie und Pädologie" inne hat und es schafft, in den verschiedenen Medien für Kinder- und Jugendmedizin ausgleichend zu wirken.

Für meine neuen Aufgaben als Präsident der Österreichischen Gesellschaft für Kinder und Jugendheilkunde gibt es viele Herausforderungen, besonders auch in der Ausbildung und Positionierung der Kinder- und Jugendmedizin (siehe auch Brief des Präsidenten: www.docs4you.at).
Die Pädiatrie hat im Bereich der medizinischen Fakultäten in Österreich in der Ausbildung an Terrain verloren, Pflichtfamulaturen sind im neuen PJ Jahr nicht vorgesehen, die Ausbildungszeit zum Arzt für Allgemeinmedizin wird aller Voraussicht ebenfalls von vier auf drei Monate reduziert werden. Daher müssen wir verschiedene Ausbildungsinhalte konsequent anbieten, das Fach Kinderund Jugendmedizin mit seinen Additivfächern und Spezialisierungen klar gegenüber der Erwachsenenmedizin darstellen. Ein wichtiger Schwerpunkt ist mit Sicherheit die Jugendmedizin und die Einführung eines Junior Checks vor der Pubertät.

Die vorliegende Ausgabe bietet eine interessante Mischung aus Schwerpunktgebieten in der Pädiatrie, mit Beiträgen zur Pulmologie, Neonatologie und Genetik und zusätzlich interessanten Informationen von Dr. Schuhmayer bezüglich ADHS und Dr. Karin Storm van's Gravesande zur Besonderheiten der MS im Kindes- und Jugendalter.

Ich wünsche „Pädiatrie und Pädologie" eine weiterhin so gute Entwicklung, die Unterstützung der ÖGKJ und auch meinerseits ist sicher.

\section{Mit freundlichen Grüßen}

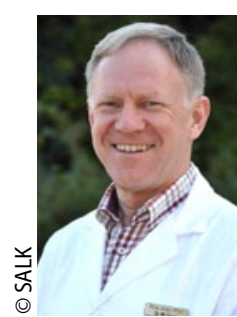

Prim. Univ.-Prof. Dr. Wolfgang Sperl Präsident der Österreichischen Gesellschaft für Kinder- und Jugendheilkunde 